\title{
JUURNAL.RU
}

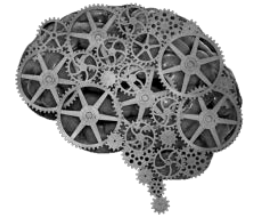

COMPANY GROUP "INTELLEKT"

\author{
Фоминых А.В. \\ Новосибирский Государственный Технический Университет (НГТУ) \\ Новосибирск, Россия
}

doi: 10.18411/lj2016-5-5-09

\section{Анализ способов мониторинга систем энергообеспечения}

Основная задача мониторинга - оперативное получение информации о состоянии объекта. Наличие системы мониторинга позволяет не только собирать и аккумулировать информацию, но и прогнозировать выход из строя узлов объекта. А это уже существенная экономия благодаря сокращению времени простоя оборудования, возможности выявить и заменить сбойный элемент на ранней стадии, не доводя дело до серьезного ремонта [1].

Цель данной работы - сформулировать рекомендации по выбору способа мониторинга системы энергообеспечения в зависимости от объекта и поставленных задач.

В этой статье рассматривается три способа работы с данными при решении задачи мониторинга.

Первым способ получения данных о работе энергосистемы - это использование плат мониторинга и счётчиков. В таком случае узнать информацию о работе энергосистемы возможно, находясь в непосредственной близости от прибора учёта. Такой подход удобен для малых нераспределённых систем или систем частного пользования. Например, информационная плата, установленная на корпус дизельгенератора для контроля уровня напряжения.

Устройства такого типа имеют ряд достоинств:

- простота реализации;

- низкая финансовая стоимость; 
- нет необходимости разработки проекта.

- К недостаткам использования плат и счётчиков можно отнести:

- отсутствие удалённого мониторинга;

- отсутствие автоматического управления;

- отсутствие архива данных;

- неудобное представление информации.

Второй способ получения данных - использование контроллера. Одним из основных преимуществ такого способа получения данных по сравнению с предыдущим является возможность удалённого мониторинга. Удалённый мониторинг повышает надежность системы за счёт оперативного получения объективной информации о состоянии объекта, при этом нет необходимости находиться в непосредственной близости от оборудования. Таким образом снижаются затраты на эксплуатацию и обслуживание, и появляется возможность быстро обнаруживать и идентифицировать неполадки или потенциально опасные нарушения в работе оборудования. Также бесспорным преимуществом такого подхода является сбор данных с нескольких устройств в одном месте.

В зависимости от поставленной задачи контроллер позволяет реализовывать автоматическое управление. Автоматическое управление позволяет мгновенно обнаруживать неполадки и значительно быстрее предпринимать действия по их устранению в сравнении с оператором.

В нынешнее время наблюдается тенденция к постепенному исчезновению аппаратных различий между компьютером и контроллером. К современным контроллерам есть возможность подключить клавиатуру, монитор и жесткий диск, а компьютеры в свою очередь приобретают признаки контроллеров: расширенный температурный диапазон, влаго- и пылезащищённость, крепление на DIN-рейку, функции самотестирования и диагностики и прочее [2].

Не смотря на применение компьютера в качестве контроллера, при использовании оборудования различных фирм невозможно создать единую 
систему мониторинга и управления. В такой ситуации приходится работать с разным программным обеспечением одновременно, что крайне удобно. Отсюда можно сделать вывод о необходимости использования третьего метода - SCADA системы.

SCADA предназначена для взаимодействия человека с автоматизированным технологическим процессом, но по сравнению с предыдущим методом она обладает рядом полезных возможностей.

К преимуществам можно отнести централизованный доступ к информации с оборудования различных производителей. Информация предоставляется в более удобном и наглядном виде, поскольку можно учесть пожелания заказчика и сделать индивидуальный интерфейс [3]. Имеется возможность быстро создавать готовые отчёты и сводки со встроенными аналитическими возможностями и инфографикой, а также вести архив данных, событий, тревог и аварий, и совмещать всё вышеперечисленное с известными базами данных. Дополнительным инструментом может быть модуль трендов, позволяющий сравнивать графики параметров и выявлять корреляцию значений, а также анализировать разницу значений в динамике [4].

Однако при всех достоинствах эти системы имеют следующие недостатки: необходимы большие финансовые затраты на покупку пакета SCADA системы и разработку проекта в ней; сложность программной реализации значительно выше и требует участия высококвалифицированных специалистов.

\section{Заключение}

По результатам работы можно предложить следующие рекомендации по выбору способа мониторинга и управления системой энергообеспечения:

Для частного использования в домах и на малых объектах разумно применять только счётчики и платы мониторинга.

Для обеспечения удалённого доступа и автоматического управления системой стоит использовать контроллер или SCADA-систему. В случаях если объект работает на оборудовании одного производителя и не требуется 
графическое отображение, целесообразно использовать контроллер. Если на объекте используется оборудование разных фирм, то возможна установка только одной SCADA-системы.

Для крупных объектов логичнее всего применять комбинированный подход к мониторингу, а именно, использование двух или более способов одновременно. Например, совместное использование контроллера и счётчиков и плат в системе мониторинга позволяет получать специализированному персоналу информацию о состоянии оборудования при проведении ремонтных и наладочных работ. Применение всех трёх способов одновременно позволит повысить надёжность и безопасность системы, то есть, данные сохранятся на контроллере при разрыве линии связи между контроллером и SCADA сервером, а при восстановлении соединения запишутся в базу данных на сервере. Таким образом, не произойдёт потери важных данных. Если произойдёт обрыв соединения между контроллером и оборудованием, то можно точно установить время аварии и вышедшее из строя узел. 


\section{Литература:}

1. Рекламно-информационный журнал «Электротехнический рынок» http://market.elec.ru/nomer/31/monitoring-sistem-elektropitaniyatelekommunikacio/ (дата обращения 29.05.2016)

2. Энциклопедия АСУ ТП

http://www.bookasutp.ru/Chapter6_1.aspx\#PLC (дата обращения 30.05.2016)

3. Средства и системы компьютерной автоматизации http://asutp.ru/?p=600658 (дата обращения 29.05.2016)

4. Отраслевой научно-технический журнал «ИСУП» http://isup.ru/articles/43/7070/ (дата обращения 30.05.2016) 